\title{
О ДИНАМИЧЕСКИХ СВОЙСТВАХ КАНОНИЗИРУЕМЫХ СИСТЕМ
}

\author{
(Представил Н. Алумяэ)
}

Продолжено исследование интегральных и локальных свойств канонизируемых (квазиканонических) систем размерности $2 n\left[{ }^{1-4}\right]$. Показано, что эти системы имеют интегральные инварианты $i$-го порядка, причем обобщенный инвариант Пуанкаре определяет квазиканоничность системы $(i=\overline{1,2 n})$. Установлено, что канонизируемые системы удовлетворяют обобщенному принципу Ливенса и модифицированному началу Гамильтона. Доказана теорема эквивалентности квазиканонической системы уравнению типа Пфаффа. Получен обобщающий случаи $\left[{ }^{3}\right]$ класс регуляторов динамических систем Лагранжа, канонизируемых лишь преобразованием времени.

1. Согласно критерию канонизируемости системы $x=X(t, x)(\operatorname{dim} x=$ $=2 n)\left[{ }^{4}\right]$, необходимо и достаточно, чтобы она имела обобщенный относительный инвариант Пуанкаре-Картана

$$
\begin{gathered}
I_{1}=\oint_{V C} \omega(\delta), d I_{1} / d s=0\left(\omega(\delta) \stackrel{\Delta}{=} b \cdot \delta x+h \delta t, b=\left(b_{i}(t, x)\right)^{*}\right), \\
b_{i}, h(t, x) \subset C_{2}(t, x), s^{*}=v^{-1}(t, x) \subset C_{1}(t, x), v \neq 0, a \cdot b=\sum_{i=1}^{2 n} a_{i} b_{i} \\
(i=\overline{1,2 n}),
\end{gathered}
$$

где $\omega(\delta)-$ пфаффиан класса $2 n+1$ с коэффициентами $b_{i}(t, x), h(t, x)$, $v(t, x)$ - произвольная, $C$ - любой замкнутый гладкий контур, пересекающий интегральную линию лишь в одной точке. Это равносильно заданию канонизируемой системы $2 n$-союзными с формой $\omega(d) \triangleq b$. - $d x+h d t$ уравнениями Пфаффа

$$
\begin{aligned}
& R x=a, x=\left(q_{1}, \ldots, q_{n}, p_{1}, \ldots, p_{n}\right)^{*}, R=\left[r_{i \sigma}(t, x)\right], x=d x / d t, \\
& r_{i \sigma}=\partial b_{i} / \partial x_{\sigma}-\partial b_{\sigma} / \partial x_{i}, a=\nabla_{x} h-b_{t}, \Delta R \triangleq \operatorname{det} R \neq 0 \quad\left(\nabla_{x} f \equiv f_{x}\right),
\end{aligned}
$$

где $\Delta R>0$ из условия на класс $\omega(d)$ и выбора независимой переменной - времени $t$.

Поэтому рассмотрение динамики систем, сводимых в канонические гладким гомеоморфизмом $\tau=T(t, x), \xi=\xi(t, x), \operatorname{dim} \xi=2 n$, проводится здесь в представлении их в виде (1.2). Во вспомогательных нормальных переменных $\tau$, $\xi$ формы $\omega\left[{ }^{4,5}\right]$ исходные уравнения и $\omega$ принимают вид

$$
\begin{gathered}
d \xi / d \tau=Z \nabla_{\xi} G, Z=\left[\begin{array}{cc}
0 & 1_{n} \\
-1_{n} & 0
\end{array}\right], \xi=\left(Q_{i}, P_{\sigma}\right)^{*}(i, \sigma=\overline{1, n}), \\
\omega=\Omega \triangleq P \cdot d Q-G(\tau, \xi) d \tau \quad\left(\Delta_{P}(G) \triangleq \Delta\left[\partial^{2} G / \partial P_{i} \partial P_{\sigma}\right] \neq 0\right) .
\end{gathered}
$$

Функция нового времени $\tau=T(t, x)$ выбрана среди нормализующих $\omega$ переменных Пфаффа из условия неинвариантности $T(t, x)$ - в рассматриваемой области нормальности $G$ по $P$ имеем $T^{*} \neq 0$. 
1.1. Йнтеградльны е с в о й ств а. Я̈сно, что любая ка́нонизируема̇я (скрытая каноническая) система имеет согласно (1.1) обобщенный универсальный инвариант Пуанкаре $I_{1}{ }^{0}=\oint_{b} \cdot \delta x\left(\delta t=0, d I_{1}{ }^{0} / d t=0\right)$, где форма $b \cdot d x-$ класса $2 n(\Delta R>0)$. Обратно, если система имеет инвариант $I_{1}{ }^{0}$ и $\Delta R \neq 0$, то $x=X(t, x)$ - канонизируемая и обладающая инвариантом $I_{1}$ система, где произвольная $h(t, x) \subset C_{2}(t, x)$, (1) - класса $2 n+1$. Переходя к переменным $\tau$, $\xi$ системы (1.3), можно аналогично $\left[{ }^{6}\right]$ показать, что всякий линейный по $\delta x$ относительный инвариант $(1.2)$ вида $I_{1^{*}}^{0}=\oint_{c} b \cdot \delta x$ обязательно пропорционален $I_{1}{ }^{0}$, т. е. $I_{1^{*}}^{0}=c_{*} I_{1}{ }^{0}, \quad$ где постоянная $c$ * не зависит от выбранного в $I_{1}^{0}, I_{1}^{0}$ * контура интегрирования $C$. С помощью формулы Стокса находим абсолютный интегральный инвариант второго порядка $I_{2}{ }^{0}$ системы (1.3)

$I_{1}^{0}=\oint_{C} b_{i} \delta x_{i}=-\int_{S} r_{i \sigma} \delta x_{i} \delta x_{\sigma}=I_{2}^{0} \quad\left(r_{i \sigma}=\frac{\partial b_{i}}{\partial x_{\sigma}}-\frac{\partial b_{\sigma}}{\partial x_{i}} ; \quad d I_{2}^{0} / d t=0\right)$,

где интеграл взят по поверхности $S$, ограниченной замкнутым $C$. Известно, что в 2n-мерном фазовом пространстве у системы (1.3), эквивалентной системе (1.2), существуют лишь следующие единственные (с точностью до постоянного множителя) универсальные относительные инварианты $I_{2 k-1}^{\prime}$ и абсолютные инварианты $I_{2 k}^{\prime}$ четных порядков

$$
\begin{aligned}
I^{\prime}{ }_{1} & =\oint_{C^{\prime}} P \cdot \delta Q=I^{\prime}{ }_{2}=\int_{S^{\prime}} \sum_{j}^{n} \delta P_{j} \delta Q_{j} \quad(j, k=\overline{1, n)}, \\
I^{\prime}{ }_{3} & =\oint_{D_{3}^{\prime}} \sum P_{j} \delta Q_{j} \delta P_{k} \delta Q_{k}=\int_{D^{\prime}} \sum \delta P_{j} \delta Q_{j} \delta P_{k} \delta Q_{k}=I^{\prime}{ }_{4}, \\
I^{\prime}{ }_{2 n-1} & =\oint_{D_{2 n-1}^{\prime}} \sum P_{j_{1}} \delta Q_{j_{1}} \ldots \delta P_{j_{n}} \delta Q_{j_{n}}=I_{2 n}^{\prime}=\int_{D_{2 n}^{\prime}} \Sigma \delta P_{j_{4}} \delta Q_{j_{1}} \ldots \delta P_{j_{n}} \delta Q_{j_{n} .} .
\end{aligned}
$$

В случае известных нормальных переменных $T(t, \xi), \xi(t, x)$ из (1.5) получим соответствующие универсальные интегральные инварианты $I_{s}{ }^{0}(2<s \leqslant 2 n)$ системы $(1.3)$, используя формулы преобразования кратных интегралов при замене переменных. Так как инварианты $I_{1}{ }^{0}$ и $I_{2 n}$ представляют наибольший интерес в исследовании динамики, найдем закон преобразования множителя $M(t, x)$ системы $x=X^{\cdot}(t, x)$ (не обязательно канонизируемой и четной размерности) при обратимой и гладкой замене переменных $\tau=\tau(t, x), y=y(t, x)(t=\theta(\tau, y)$, $\left.x=\xi(\tau, y) ; \tau, y \subset C_{\perp}(t, x)\right), \quad$ приводящей исходную к $d y / d \tau=\mathcal{Y}(\tau, y)$ со множителем $N(\tau, y)$. Примем обозначения

$$
\begin{aligned}
& \lambda=\lambda(t, x)=\tau ;, Y=y ; y=\lambda^{-1} Y\left(Y=Y(t, y), f=\left(\frac{\partial}{\partial t}+X_{s} \frac{\partial}{\partial x_{s}}\right) \circ f\right), \\
& \lambda^{*}(t, y)=\lambda(t, x(t, y))=\tilde{\lambda}(\tau, y) \triangleq \lambda(\theta(\cdot), \xi(\cdot))=\lambda(t, x)(s=\overline{1, m}) .
\end{aligned}
$$

Если функция $N(\tau, y)$ - множитель (1.6), то необходимо и достаточно, чтобы она удовлетворяла уравнению

$$
d N / d \tau+N \underset{y}{\operatorname{div}} \tau^{\prime}(\tau, y)=0 \quad\left(M_{t}+\operatorname{div}_{x} M X=0\right) .
$$

С учетом (1.6) подстановкой в (1.7) находим множитель $N$ в виде 


$$
\begin{gathered}
N=\hat{\lambda} \theta_{\tau=\tau *} \Delta^{-1} \tilde{M}, \tilde{M}(\tau, y) \stackrel{\Delta}{=} M(\theta(\cdot), \xi(\cdot)), \Delta=\operatorname{det}[\partial y / \partial x]_{x \rightarrow y}^{t+* \bar{t}}, \\
\theta_{\tau=\tau^{*}}=\left.(\partial \theta / \partial \tau)\right|_{\tau=\tau^{*}}=\left(\partial \tau^{*} / \partial t\right)^{-1} \\
\left(\tau^{*}(t, y)=\tau(t, x(t, y)) ; \tau^{*}(0, y)=0, \tau(0, x)=0\right) .
\end{gathered}
$$

Если $\tau(0, x) \equiv 0$, то согласно (1.6), (1.7) находим

$$
\begin{gathered}
\tau^{*}(t, y)=\int_{0}^{t} \lambda^{*}\left[\sigma, y\left(\sigma, y^{0}\right)\right] d \sigma\left(y^{*}=Y, y=y\left(t, y^{0}\right), y^{0} \triangleq y\left(0, y^{0}\right)\right), \\
\tau_{t}^{*}=\lambda^{*}\left(0, y^{0}\right)+\int_{0}^{t} \lambda_{t}^{*} d \sigma .
\end{gathered}
$$

Отсюда при $\lambda_{t}{ }^{*} \equiv 0$ следует, что $\tau_{t}{ }^{*}-$ инвариант преобразованной системы, ибо $\lambda^{*}\left(0, y^{0}\right)$ - ее инвариант. В этом случае $N^{*}=\tilde{\lambda} \Delta^{-1} \tilde{M}-$ множитель преобразованной системы. Обобщим рассмотренный случай.

Л ем м а. Пусть $f(t, x)$ удовлетворяет уравнению $D f / D t=w(x)\left(w_{t} \equiv 0\right)$, $\partial X_{s} / \partial t \equiv 0 \quad\left(D / D t=\partial / \partial t+X_{s} \partial / \partial x_{s}\right) . \quad$ Тогда $f_{t}-$ инвариант исходной $(и$ преобразованной) системы $(s=\overline{1, m})$.

Действительно, ввиду $m$ последних условий операторы $\partial / \partial t$ и $D / D t$ коммутативны: $\partial / \partial t \circ D / D t=D / D t \circ \partial / \partial t$. Дифференцируя это уравнение по $t$ частным образом с учетом коммутативности, получим равенство $D f_{t} / D t=0$, доказывающее лемму. Используя ее и равенства $\tau^{*}=\lambda$, $\lambda_{t}{ }^{*} \equiv 0, Y_{t} \equiv 0$, замечаем, что предыдущий результат сохраняется при $\tau(0, x) \not 0$. Вид связи $t, \tau$ не требуется, если преобразование $x \rightarrow y$ задано решением $x_{1}=\varphi(t, y)$ системы уравнений $x=Z(t, x)$ и выполнены условия $\partial \tilde{\lambda} / \partial \tau=0, \partial \tilde{Y} / \partial \tau=0, \partial \tilde{M} / \partial \tau=0, \Lambda_{0} \triangleq \operatorname{div} Z=0$. Из $\tilde{\lambda}_{\tau}=0$,

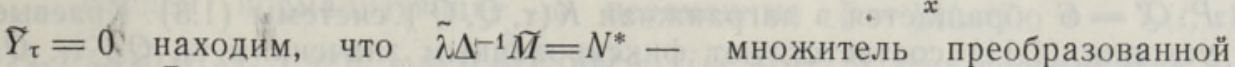
системы. В силу $\Lambda_{0}=0$ и уравнения $\Delta_{t}{ }^{0}=\Lambda_{0} \Delta^{0}$ имеем $\Delta^{0}=\Delta^{0}(y)=$ $=\operatorname{det}[\partial \varphi / \partial y]=\Delta^{-1}$, т. е. $\Delta \triangleq \operatorname{det}[\partial y / \partial x]=\Delta(y)$. Отсюда $N^{*}$ не зависит явно от связи $t, \tau$ при $x=\varphi(t, y)$ и принятых условиях на $\tilde{\lambda}, \tilde{Y}, \tilde{M}, Z$ $(\tilde{f}=f(\theta(\tau, y), \xi(\tau, y))$. С учетом обозначений (1.6) система (1.2), эквивалентная (1.3) с $N=1$, имеет согласно (1.8) интегральный инвариант $I_{2 n}$ и множитель вида

$$
I_{2 n}=\int_{D_{2 n}} M(t, x) \delta x_{1} \ldots \delta x_{2 n}, M(t, x)=\lambda^{-1} \tau_{t}^{*} \Delta .
$$

1.2. Обобщенны й принци п Ли в нс а. Решения (1.2) являются экстремалями линейного по $\tilde{x}^{\cdot}$ функционала Пфаффа

$$
J[\tilde{x}]=\int_{t_{0}}^{t_{1}}(b \cdot \tilde{x}+h) d \sigma\left(\tilde{x}=\left(\tilde{x}_{1}, \ldots, \tilde{x}_{2 n}\right)^{*}=\left(\tilde{q}_{1}, \ldots, \tilde{p}_{n}\right)^{*}\right)
$$

в семействе линий $\tilde{x}[t] \subset C_{1}\left(t^{0}, t^{1}\right)$ с концами на $2(n+1)$-поверхностях $T\left(t_{0}, \tilde{x}\left(t_{0}\right)\right)=\tau_{0}, Q\left(t_{0}, \tilde{x}\left(t_{0}\right)\right)=Q_{0} ; T\left(t_{1}, \tilde{x}\left(t_{1}\right)\right)=\tau_{1}, Q\left(t_{1}, \tilde{x}\left(t_{1}\right)\right)=Q_{1},(1.11)$

где $Q_{j}=\left(Q_{j}(t, x)\right)^{*}, j=\overline{1, n} ; \tau_{0}, Q_{0}, \tau_{1}, Q_{1}$ - фиксированные постоянные.

Вычисляя при (1.11) вариацию (1.10) на решении $x(t)$ системы (1.2) и учитывая $\omega=\Omega$, получим равенства

$$
\mathcal{E}_{\tilde{x}} \circ f_{0}=0, f_{0}=b(t, \tilde{x}) \cdot \tilde{x}+h(t, \tilde{x}), \mathcal{E}_{\tilde{x}}=\nabla_{\tilde{x}}-\frac{d}{d t} \circ \nabla_{\tilde{x}} .
$$




$$
\delta J[x]=[b(t, x(t)) \cdot \delta x+h(t, x(t)) \delta t]_{0}^{1}=[P \cdot \delta Q-G(\tau, \xi(\tau)) \delta \tau]_{0}^{1}=0,
$$

доказывающие утверждение. Система (1.2) имеет вариационную форму Әйлера для $J$. Расширение допустимого класса сравнения $\{\tilde{x}(t)\}$ (обычно с фиксированными концами для (1.3)) получено за счет применения части $\tau, Q_{j}$ нормализующих переменных. Заметим, что принцип Ливенса для (1.3) эквивалентен началу Гамильтона с лагранжианом $K\left(\tau, Q, Q^{\prime}\right) \triangleq P \cdot Q^{\prime}-G\left(Q^{\prime}=\partial G / \partial P\right)$ лишь при фиксированных $\tau_{0}$, $Q_{0}, \tau_{1}, Q_{1}$. Аналогично $\left[{ }^{7,8}\right]$ решения (1.2) можно интерпретировать как экстремали условной задачи Лагранжа в форме модификации начала Гамильтона.

1.3. Модифицированны й принцип Гамильтона. Из обобщенного принципа Ливенса $(1.10)$ - (1.12), используя вспомогательные переменные $\tau$, $\xi$ и результат $\left[{ }^{7,8}\right]$, находим, что система (1.2) определяется следуюшим модифицированным принципом Гамильтона и удовлетворяет ему. Решения (1.2) - экстремали задачи Лагранжа $\delta J[x]=0$ при краевых условиях (1.11) и $n$ линейных дифференциальных связях вида

$$
\begin{aligned}
& l_{j}(t, x \mid x) \triangleq \partial Q_{j} / \partial t+x_{s} \partial Q_{j} / \partial x_{s}-g_{j}\left(\partial T / \partial t+x_{s} \partial T / \partial x_{s}\right)=0 \\
& \left(l_{j}=Q_{j}-g_{j} T=0, g_{j}(t, x) \stackrel{\Delta}{=} \partial G / \partial P_{j} ; j=\overline{1, n} ; s=\overline{1,2 n)} .\right.
\end{aligned}
$$

Дифференциальные условия (1.13) - независимые линейные комбинации уравнений $(1.2)$, где $l_{j}=\lambda_{j s} \mathcal{E}_{x s} \circ f_{0}, \partial \lambda_{j s} / \partial x_{\sigma} \equiv 0(s, \sigma=\overline{1,2 n})$. Уравнения $l_{j}\left(\cdot \mid x^{*}\right)=0$ относительно $x_{s}^{*}$ имеют ранг $n$. В пространстве переменных $t, x, x$ они представляют многообразие стационарности $Q^{\prime}=\partial G / \partial P$ по $\widetilde{P}[\tau]$ действия Ливенса для (1.3), где ядро функционала $P \cdot Q^{\prime}-G$ обращается в лагранжиан $K\left(\tau, Q, Q^{\prime}\right)$ системы (1.3). Краевые условия (1.11) соответствуют фиксированным значениям $\tau_{0}, Q_{0}, \tau_{1}, Q_{1}$ принципа Ливенса для (1.3). Из $\omega=\Omega$ при известных $\tau=T(t, x)$, $\xi=\xi(t, x)$ находим на связях (1.13) значение ядра $f_{0} \rightarrow f_{0}{ }^{*}$ в виде линейной функции от $x_{s}$

$f_{0}^{*}=\left.(b \cdot x+h)\right|_{l=0}=\left.(d T / d t)\right|_{l=0} \cdot K\left(T, Q, Q^{\prime}\right), l=\left(l_{j}\right)^{*}, \tau=T(t, x)$,

где $Q^{\prime}=G_{P}=g(t, x),\left(P \cdot Q^{\prime}-G\right) d T=(b \cdot x+h) d t \quad(j, \sigma=\overline{1, n})$.

В силу равенсте, эквивалентных условиям нормализации $\omega$ в $\tau$,

$$
P_{\sigma} \partial Q_{\sigma} / \partial x_{i}-G \partial T / \partial x_{i}=b_{i}, \quad P_{\sigma} \partial Q_{\sigma} / \partial t-G \partial T / \partial t=h \quad(i=\overline{1,2 n})
$$

с заданной нормальной по $P$ функцией $G$, величина $T$ принимает вид $T^{*}=G^{-1}\left[P_{j} \partial Q_{j} / \partial t+x_{s} P_{j} \partial Q_{j} / \partial x_{s}-\left(b_{s} x_{s}+h\right)\right](\partial T \cdot \partial \tau=0$ при $\partial G / \partial \tau=0)$. Если заменить $T \cdot$ при $l=0$ на ее значение из (1.2), то согласно (1.14) $f_{0}{ }^{*}$ будет функцией лишь от $t, x$. Этот вариант обобщает рассмотренные в $\left[{ }^{3}\right]$ упрощешные случаи $p=L_{q}, b_{v}=0(v=\overline{n+1,2 n})$.

2. Локальные свойства. Теорем э эвивалентности. Н еобходи мость. Рассматривая вариацию функционала

$$
J\left[\tilde{x} \mid t, t_{0}, x_{0}\right]=\int_{t_{0}}^{t}(b \cdot \tilde{x}+h) d t
$$

на экстремалях $x=f\left(t, x_{0}\right)$, определенных системой (1.2), найдем 


$$
\delta J=\delta \tilde{S}\left(t, t_{0}, x_{0}\right)=\left.(b \cdot \delta x+h \delta t)\right|_{t_{\mathrm{v}}} ^{t}\left(S=J[f \mid \cdot], x_{0} \equiv f\left(t, x_{0}\right)\right) .
$$

Фиксируя $t_{0}$ и обозначая $S=\left.\widetilde{S}\right|_{t_{0}=\text { fixconst }}=S\left(t, x_{0}\right)$, из равенств (2.1) и $d S=\left.\delta J\right|_{t_{0}=f i x c o n s t}$ получим, что решения $f\left(t, x_{0}\right)$ должны иметь локальное свойство, определенное уравнением типа Пфаффа на вектор-функцию $f\left(t, x_{0}\right)$

$$
b\left(t, f\left(t, x_{0}\right)\right) \cdot d f+h(t, f(\cdot)) d t=b\left(t_{0}, x_{0}\right) \cdot d x_{0}+d S\left(t, x_{0}\right),
$$

которое она обращает в тождество по $t$, $x_{0}$. Необходимость $(2.2)$ доказана.

Переходя к $\tau$,, , получим для соответствующих решений $\xi=\varphi\left(\tau, \xi_{0}\right)$ ассоциированной канонической системы (1.3) равенства

$$
\begin{aligned}
& b(t, f) \cdot d f+h(\cdot) d t--d S=P \cdot d Q-G(\tau, \varphi(\cdot)) d \tau-d \Psi\left(\tau, \xi_{0}\right), \\
& S\left(t, x_{0}\right)=\Psi\left(\tau, \xi_{0}\right)=\int_{\tau_{0}}^{\tau} K\left(\tau, Q, Q^{\prime}\right) d \tau \quad\left(d S=d \Psi, P=\partial K / \partial Q^{\prime}\right),
\end{aligned}
$$

$b_{0} \cdot d x_{0}=P_{0} \cdot d Q_{0} \quad\left(K=P \cdot Q^{\prime}-G=(b \cdot x+h) d t / d \tau, \quad \tau_{0}=\tau\left(t_{0}, x_{0}\right)=\right.$ fixconst $)$,

$$
\xi_{0}=\varphi\left(\tau_{0}, \xi_{0}\right), b_{0} \triangleq b\left(t_{0}, x_{0}\right), \xi=\varphi\left(\tau, \xi_{0}\right) \leftrightarrow x=f\left(t, x_{0}\right), \xi_{0} \leftrightarrow x_{0} .
$$

Д ост а точность. Докажем обратное утверждение.

Пусть существуют непрерывно дифференцируемые функции $b_{s}(t, x)$, $h(t, x)$ - коэффициенты формы $\omega$ класса $2 n+1$ и заданы функции $F\left(t, x_{0}\right), F_{t}\left(t_{0}, x_{0}\right) \subset C_{1}(\zeta \times \delta) \quad\left(\delta=\left\{x_{0}\right\}-\right.$ область $)$, где $\Delta\left[\partial F / \partial x_{0}\right] \neq 0$ $\left(F\left(t_{0}, x_{0}\right) \equiv x_{0}\right)$, приводящие $\omega$ к виду

$$
\omega=b\left(t_{0}, x_{0}\right) \cdot d x_{0}+d V\left(t, x_{0}\right)\left(V \subset C_{2}\left(t, x_{0}\right), b_{t} \equiv 0\right) .
$$

Тогда $F\left(t, x_{0}\right)$ - решения $f$ союзной с $\omega$ системы Пфаффа (1.2).

Введем нормализующие $\omega$ переменные $\xi=\xi(t, x), \quad \tau=T(t, x)$, $\xi, T \subset C_{1}(t, x), \tau \neq 0, \omega=\Omega \triangleq P \cdot d Q-G(\tau, \xi) d \tau$. В этих координатах линии $x=F\left(t, x_{0}\right)$ соответствует кривая $\xi=\Phi\left(\tau, \xi_{0}\right), \Phi\left(\tau_{0}, \xi_{0}\right) \equiv \xi_{0}$, a функция $V\left(t, x_{0}\right)$ переходит в $W\left(\tau, \xi_{0}\right)=V\left[\theta\left(\tau, \Phi\left(\tau, \xi_{0}\right), x_{0}\left(\xi_{0}\right)\right](t=\right.$ $=\theta(\tau, \xi))$. Фиксируя $t=t_{0}$ и, соответственно, $\tau=\tau_{0}$, аналогично (2.3) имеем

$$
b(t, F) \cdot d F+h(t, F) d t=P \cdot d Q-G(\tau, \Phi) d \tau ; d V=d W, b_{0} \cdot d x_{0}=P_{0} \cdot d Q_{0} .
$$

Согласно условиям (2.4), функции $Ф\left(\tau, \xi_{0}\right), W\left(\tau, \xi_{0}\right)$ имеют необходимую гладкость на образе $\boldsymbol{\zeta}^{\prime} \times \delta^{\prime}$ произведения $\mathfrak{\zeta} \times \delta$, где $\Delta\left[\partial \Phi / \partial \xi_{0}\right] \neq 0$ $\left(W\left(\tau, \xi_{0}\right) \subset C_{2}, G(\tau, \xi) \subset C_{1}\right)$. В силу $(2.4),(2.5)$ они удовлетворяют уравнению

$$
P \cdot d Q-G(\tau, \Phi(\cdot)) d \tau=P_{0} \cdot d Q_{0}+d W\left(\tau, \xi_{0}\right) \quad\left(\xi=\Phi\left(\tau, \xi_{0}\right)\right),
$$

где правая часть содержит $\tau$ лишь в $d W$. Из теоремы эквивалентности $\left[{ }^{8}\right]$ для (1.3) заключаем, что $\Phi\left(\tau, \xi_{0}\right)-$ решения союзной с $\Omega$ системы (1.3). Поэтому $F\left(t, x_{0}\right)$ - решения (1.2). Достаточность доказана. Поэтому (1.2) удовлетворяет следующей модификации теоремы эквивалентности.

Т ео рем а. Система (1.2) эквивалентна при условиях (2.4) уравнению (2.2) с правой частью, зависящей от $t$ лишь в последнем члене.

Этот результат обобщается аналогично $\left[{ }^{8}\right]$ на случай решений $(1.2)$, данных уравнениями $x=g\left(t, \gamma_{0}\right)$, где независимые параметры $\gamma_{0}$ задают траектории (1.2). Для этого достаточно сменить пбозначения в соотношениях $(2.2)-(2.6)$, после чего форма $b_{0} \cdot d x_{0}+d S\left(t, x_{0}\right)$ примет вид $\beta\left(t_{0}, \gamma_{0}\right) \cdot d \gamma_{0}+d R\left(t, \gamma_{0}\right)$. Достаточность главного условия $(2.2)$ 
можно обосновать непосред̈ственной проверкой (без перехода к $\xi, \tau$ и результата $\left.\left[{ }^{8}\right]\right)$.

3. Пр и меры. Найдем класс канонизируемых лишь преобразованием времени $t=\theta\left(\tau, q, q^{\prime}\right)$ и управляемых силами fo систем Лагранжа вида (1.2)

$q^{\cdot}=H_{p}, p^{\cdot}=-H_{q}+f^{0}\left(f^{0}, H \subset C_{2}(t, x), \Delta_{p}(H) \neq 0, q^{*}=\frac{d q}{d t}, q^{\prime}=\frac{d q}{d \tau}\right)$,

$$
\begin{gathered}
q=Q, t=\theta\left(\tau, q, q^{\prime}\right), q=\hat{\lambda}\left(\tau, q, q^{\prime}\right) q^{\prime} \\
\left(\Delta=\hat{\lambda}^{n-1} \Delta_{1} \neq 0, \hat{\lambda}=\tau, p=L_{q} \cdot L=p \cdot q \cdot H\right),
\end{gathered}
$$

$\hat{\lambda} \neq 0, \Delta_{1}=\theta_{\tau}\left(\hat{\lambda}+q^{\prime} \cdot \hat{\lambda}_{q^{\prime}}\right)-\hat{\lambda}_{\tau}\left(q^{\prime} \cdot \theta_{q^{\prime}}\right) \quad\left(\hat{\lambda}^{-1}=\theta_{\tau}+q^{\prime} \cdot \theta_{q}+q^{\prime \prime} \cdot \theta_{q^{\prime}}, f_{x}=\partial f / \partial x\right)$, где $\theta$ определена производной $d \theta / d \tau=\hat{\lambda}^{-1}\left(\tau, q, q^{\prime}\right)$ в силу системы

$$
\begin{gathered}
q^{\prime}=G_{P}, P^{\prime}=-G_{q} \\
\left(P=K_{q^{\prime}}, K\left(\tau, q, q^{\prime}\right)=P \cdot q^{\prime}-G, G(\tau, q, P) \subset C_{2} ; \Delta_{P} G \neq 0\right),
\end{gathered}
$$

удовлетворяющей принципу Гамильтона. В нее по условию сводится система (3.1) обратимым преобразованием при $\Delta \neq 0$. Функция $G$ не обязательно подобна $H(G(T, q, P) \not \equiv H(t, q, p))$. Обозначим

$$
\begin{gathered}
\lambda\left(\tau, q, q^{*}\right)=\hat{\lambda}\left(\tau, q, q^{\prime}\right)=\tilde{\lambda}(\tau, q, P), \Delta^{0}=-\mathcal{G}[\lambda], \Delta_{0}=\hat{\lambda}+q^{\prime} \cdot \hat{\lambda}_{q^{\prime}} \neq 0, \\
0 \neq \Delta_{0} \leftrightarrow \Delta^{0} \neq 0, \Theta[f]=q \cdot f_{q^{*}}-f,
\end{gathered}
$$

$$
\vartheta\left(\tau, q, q^{*}\right)=\theta\left(\tau, q, q^{\prime}\right)=\tilde{\theta}(\tau, q, P)=t,
$$

$\tau=T\left(t, q, q^{*}\right), M\left(\tau, q, q^{*}\right)=K\left(\tau, q, \lambda^{-1} q^{*}\right) \not \equiv L\left(t, q, q^{*}\right), N=\lambda M\left(\Delta=\lambda^{n+1} \vartheta_{\tau} / \Delta^{0}\right)$.

Искомый класс зададим множеством $\left\{f^{0}\right\}$ всех регуляторов $f^{0}(t, x)$, при которых исходная система переходит в (3.2) преобразованием (3.1). Сперва покажем, что искомая система удовлетворяет при $\Delta^{0} \neq 0$ модифицированному принципу Гамильтона (раздел 2), в котором решения (3.1) - экстремали функционала $J_{1}$ при условии $\tau^{*}=\lambda$, свободных $t_{0}, t_{1}$ и фиксированных значениях

$\tilde{q}\left[t_{0}\right]=q_{0}, \tilde{q}\left[t_{1}\right]=q_{1}, \tau\left[t_{0}\right]=T\left(t, q_{0}, q_{0}^{*}\right)=\tau_{0}, \tau\left[t_{1}\right]=T\left(t_{1}, q_{1}, q_{1}^{*}\right)=\tau_{1}$,

где $J_{1}=\int_{t_{0}}^{t_{1}} N\left(\tau, q, q^{*}\right) d t, \delta J_{1}=0, N=\lambda K, q=\lambda q^{\prime}$.

Вводя множитель $v(t)$, импульс $\psi$ и функции $\varphi, g$ вида

$$
\varphi=\lambda(M-v(t)), \psi=\varphi_{q}, g(\tau, q, \psi, v(t))=\psi \cdot q^{*}-\varphi,
$$

представим экстремали в канонической форме

$$
q=g_{\psi}, \psi=-g_{q}, \tau=g_{v}=\lambda, v=-g_{\tau}
$$

с краевыми условиями (3.4) и условием трансверсальности по $t$

$$
g\left(\tau_{1}, q_{1}, \psi_{1}, v\left(t_{1}\right)\right)=g\left(\tau_{0}, q_{0}, \psi_{0}, v\left(t_{0}\right)\right)=0
$$

Так как из (3.5), (3.6) имеем $g^{*}=g_{t}, g_{t} \equiv 0$, то согласно (3.7) $g[t] \equiv 0$. С учетом (3.3) $v=v^{0} \triangleq \mathcal{G}[N] / \mathcal{G}[\lambda]$. Введем функцию $V(\tau, q, \psi)$ уравнением $g(\tau, q, \psi, V)=0$. Дифференцируя тождество $g(\cdot, V(\cdot)) \equiv 0$ по $q, \psi$, найдем, что экстремали (3.4) - (3.7) удовлетворяют системе 


$$
q=-\lambda V_{\psi}, \quad \psi=\lambda V_{q} .
$$

Из (3.2), (3.6) и значения $v=v^{0}$ получим равенства

$$
\begin{gathered}
V=M-\lambda^{-1} \psi \cdot q^{*}, \quad \psi=P+\left[K-V-\left(q^{\prime} \cdot P\right)\right] \lambda_{q}, \\
\Delta^{0} \lambda^{-1}\left[q^{\prime} \cdot P-K+V\right]=0 \quad\left(\Delta^{0} \neq 0\right),
\end{gathered}
$$

эквивалентные $V=-G, \psi=P$. Отсюда из (3.2), (3.8) следует стационарность $J_{1}$ при $(3.4), \tau^{*}=\lambda$ на решениях системы $(3.1)$, сводимой к (3.2) лишь преобразованием $t \rightarrow \tau(q \equiv Q)$. Совокупность искомых регуляторов $f^{0}(t, x)$ зависит от функций $T, \lambda, K, L$ и выражается равенствами

$$
f^{0}=-L_{q}+L_{(1)} q^{*}+l_{(1)}+\lambda^{2} L_{(0)} C\left(k_{(2)}-\lambda^{-1} K_{(1)} q^{*}-k_{(1)}\right)-1-\tilde{\lambda}^{\prime} L_{(0)} q ;,
$$

$$
L_{(1)}=\left[\partial^{2} L / \partial q_{i}^{*} \partial q_{s}\right], L_{0}=\left[\partial^{2} L / \partial q_{i}^{*} \partial q_{\sigma}^{*}\right], l_{(1)}=\partial^{2} L / \partial t \partial q^{*}, \tau=T\left(t, q, q^{*}\right),
$$

$C=\left[\partial^{2} G / \partial P_{\sigma} \partial P_{s}\right]=A^{-1}, A=\left[\partial^{2} K / \partial q_{\sigma}^{\prime} \partial q_{s}^{\prime}\right], \Delta A \neq 0 \quad(i, \sigma, s=\overline{1, n})$,

$$
K_{(1)}=\left[\partial^{2} K / \partial q_{s}^{\prime} \partial q_{\rho}\right], \quad k_{(2)}=\left[\partial K / \partial q_{s}\right]^{*}, \quad k_{(1)}=\left[\partial^{2} K / \partial \tau \partial q_{s}^{\prime}\right]^{*}\left(q^{\prime}=\lambda^{-1} q^{*}\right),
$$

где $T$ - решение уравнения $\vartheta(\tau, \cdot)=t$ при $\Delta \neq 0, \Delta^{0} \neq 0$, а остальные обозначения имеют смысл (3.1), (3.3). Подставляя $\tau=T\left(t, q, q^{*}\right)$ в (3.9), находим $\left\{f^{0}\right\}$ как вектор-функцию от $t, q, q$. Задавая $\tilde{\lambda}, \theta$, можно рассматривать связь $(\tilde{\lambda})^{-1}=\tilde{\theta}_{\tau}+(\tilde{\theta}, G)$ как уравнение для определения нормальной $G(\tau, q, P)$. Полученный класс обобщает рассмотренные в $\left[{ }^{3}\right]$ случаи, где функция $T$ явно не фигурирует.

3.1. С истем а Р ауса. Рассмотрим натуральную систему Лагранжа (3.1) при $r$ независимых линейных идеальных неголономных связях $N q+v=0$, взятых в разрешенном по ${ }^{*}$ виде

$$
\begin{gathered}
\zeta=V \eta+v, V=\left[v_{\alpha^{\prime} j}(t, q)\right], v=\left(v_{\alpha^{\prime}}(t, q)\right)^{*} \subset C_{1}(t, q), \\
q=\left[\begin{array}{l}
\eta \\
\zeta
\end{array}\right], p=\left[\begin{array}{c}
y \\
z
\end{array}\right], \eta=\left(q_{j}\right)^{*}, \zeta=\left(q_{\alpha}\right)^{*}, y=\left(p_{j}\right)^{*}, z=\left(p_{\alpha}\right)^{*}, \\
j=\overline{1, l,} \overline{\alpha=l+1, m}, \\
r=m-l=\operatorname{dim} z=\operatorname{dim} \zeta, N_{q}=-v ; \alpha^{\prime}=\alpha-l=\overline{1, r}, \\
N=\left[V,-\mathbf{1}_{r}^{r}\right]-(r \times m) \text {-матрица, } V-(r \times l) \text {-матрица } \\
H=1 / 2 p \cdot H_{2} p+\gamma \cdot y+k \cdot z+H_{0}(t, q)\left(H_{2}, \gamma, k \subset C_{1}(t, q)\right) .
\end{gathered}
$$

Запишем уравнения движения этой системы с вектором множителей $\lambda(t)$ Payca

$$
\begin{gathered}
y \cdot=V^{*} \lambda-H_{\eta}, q=H_{p} \quad\left(p=-H_{q}+N^{*} \lambda\right), \\
z^{*}=-H_{\zeta}-\lambda, H_{z}=V H_{y}+v, 2 H_{2} \triangleq y \cdot A y+z \cdot C z+2 B y \cdot z, \\
H_{2}=\left[\begin{array}{ll}
A & B^{*} \\
B & C
\end{array}\right]=\left[h_{i k}(t, q)\right]>0 ; i, k=\overline{1, m}=\operatorname{dim} q=\operatorname{dim} p, \\
A-(l \times l)-, \quad B-(r \times l)-, \quad C-(r \times r) \text {-матрицы. }
\end{gathered}
$$

Исключим из (3.11) вектор $\lambda(t)$. Тогда из (3.11) с учетом (3.10) имеем

$$
\left(C-V B^{*}\right) z=(V A-B) y+V \gamma+v-k .
$$

Ограничимся системами Рауса, где 


$$
\Delta_{0} \neq 0 \quad\left(\Delta_{0} \triangleq \operatorname{det} D_{0}, D_{0}=C-V B^{*}\right) .
$$

Согласно (3.12), (3.13) $z$ будет линейной функцией $y$ вида

$$
\left.z=z_{0}(t, q \mid y) \triangleq Z=Z y+w \stackrel{\Delta}{=} D_{0}^{-1}(V A-B), w=D_{0}^{-1}(V \gamma+v-k)\right) .
$$

Дифференцируя (3.14), из (3.10), (3.11) находим вектор-функцию

$$
\begin{gathered}
\lambda=D_{1}^{-1}\left\{(V A-B) H_{\eta}-D_{0}\left(H_{\zeta}+Z \cdot y+w\right)\right\}_{0}=\lambda_{0}(t, q, y) \quad\left(\lambda=\left(\lambda_{1}, \ldots, \lambda_{r}\right)^{*}\right), \\
D_{1}=D_{0}+(V A-B) V^{*}, Z \cdot=\left.Z_{0}(t, q, y) \triangleq(d Z / d t)\right|_{q^{*}}=(\partial H / \partial p)_{0}, \\
w=w_{t}+w_{q} \cdot(\partial H / \partial p)_{0} \triangleq w_{0}(t, q, y) \quad\left((\partial H / \partial p)_{0}=\left.(\partial H / \partial p)\right|_{z=z_{\alpha}(t, q \mid y)}\right) .
\end{gathered}
$$

Нижний индекс «нуль» обозначает подстановку (3.14), (3.15) в (3.11) с учетом (3.10). При этом $\Delta_{1} \triangleq \operatorname{det} D_{1} \neq 0$ в (3.15). Действительно, из положительности квадрики $2 \tilde{\mathscr{H}}_{2}$ на $y+V^{*} z=0, z \neq 0,2 \tilde{\mathscr{J}}_{2} \neq 0$, где она вида $z: D_{1} z>0$, следует $\Delta_{1} \neq 0$. Подставляя (3.14), (3.15) в (3.11), найдем ее замкнутую подсистему

$$
q=\left(H_{p}\right)_{0}, \quad y=-\left(H_{\eta}\right)_{0}+V^{*} \lambda_{0}(t, q, y)
$$

размерности $2 m-r$, четного порядка при $r=2 \varrho<m$ (@-целое). В последнем случае при условиях (3.10), (3.13) канонизируемая система (3.16) имеет все рассмотренные в разделах 1 и 2 динамические свойства.

Заметим, исходя из (1.2), что для канонизируемости системы $x=X(t, x)(\operatorname{dim} x=2 \mathrm{Q})$ необходимо и достаточно, чтобы ее правая часть $X(t, x)$ была представима в виде $R^{-1} a$. Если же $X(t, x)$ задана, то для квазиканоничности этой системы достаточно, чтобы существовало решение системы 2Q-линейных однородных уравнений в частных производных $R X=a$ относительно коэффициентов формы $\omega$ при условиях (1.1) и $\Delta R \neq 0$.

\section{ЛИТЕРА Т У РА}

1. Чаплыгин С. А. Исследования по дннамике неголономных систем. М.-Л., ГИТТЛ, 1941 , c. $28-38$.

2. Биркгоф Дж. Д. Динамические системы. М.-Л., ОГИЗ, ГИТТЛ, 1941, с. 34-54.

3. Кейс И. Изв. АН ЭССР, Физ. Матем., 29, № 4, 439-441 (1980).

4. Кейс И. Изв. АН ЭССР, Физ. Матем., 30, № 2, 109-116 (1981).

5. Рашевский П. К. Геометрическая теория уравнений с частными производными. М.-Л., ОГИЗ, Гостехиздат, 1947, с. 142-168.

6. Hwa Chung Lee. Proc. Roy. Soc. Edinburgh A, LXII, 237-247 (1947).

7. Гельфанд И М., Фомин С. В. Вариационное исчисление. М., ГИФМЛ, 1961, с. $71-$ $78,210-228$.

8. Парс Л. Аналитическая динамика. М., «Наука», 1971, с. 282-290.

Ннститут кибернетики Академии наук Эстонской ССР
Поступила в редакцию 5 августа 1982 


\section{KANONISEERITAVATE SUSTEEMIDE DUNAAMILISTEST OMADUSTEST}

Artiklis on analüüsitud $2 n$-järguliste kanoniseeritavate süsteemide globaalseid ja lokaalseid omadusi. On saadud erinevad Poincaré-Cartani tüüpi integraalsed invariandid $I_{m}$ (1.5) globaalsete dünaamiliste omaduste kirjeldamiseks $(m=1,2 n)$, leitud Jacobi kordaja teisenduse valem (1.8) vastavalt kõigi muutujate (1.6) transformatsioonile ning esitatud üldistatud Livensi (1.11), (1.12) ning modifitseeritud Hamiltoni (1.11), (1.13) printsiibid kanoniseeritavate süsteemide jaoks. On tõestatud algsüsteemi ja Pfaffi võrrandi ekvivalentsuse teoreem kanoniseeritavuse puhul. Näitena on vaadeldud kanoniseeritavate süsteemide $(3.1),(3.10)$ spetsiaalseid omadusi.

\section{KEIS}

\section{ON THE DYNAMICAL PROPERTIES OF THE SYSTEMS REDUCIBLE TO THE CANONICAL FORM}

The global and the local properties of the systems noted in the headline, are investigated in this paper. Account is taken of the two basic ideas. The first one developes an analogy between a canonical and a quasi-canonical system, the second treats every quasi-canonical system as associated with the Pfaffian of the $2 n+1$ rank, due to the relevant criterion in $\left[{ }^{4}\right]$. On this ground the systems (1.2) obtain relative integral invariants $I_{2 k-1}$ of the odd order and absolute integral invariants $I_{2 k}$ of the even order (see formulae (1.5)). Hence, the universal Poincaré-type invariant

$$
I_{1}^{10}=\oint_{\mathrm{V} C} b \cdot \delta x, \quad d I_{1}^{0} / d t=0 \quad(\text { see }(1.1))
$$

defines the reducibility to the canonical form $(k=1, n)$.

The Jacobi multiplier transformation rule, covering the general case (1.6) with the time substitution, is derived from (1.8). Under conditions (1.11), (1.13) the modified Hamilton and the generalized Livens principles are proposed.

The essential local properties relevant to the treated systems are established in the (2.2) statement.

As an example, the Lagrange and the Routh quasi-canonical systems are considered. The class of regulators (3.9) corresponding to the time transform (3.1) is obtained. 